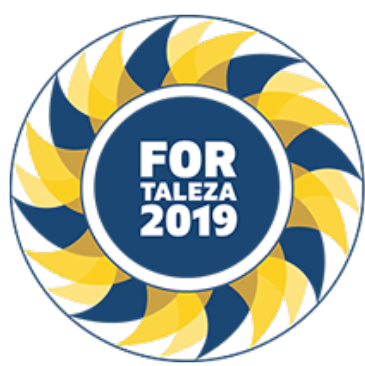

\title{
EVALUATION OF ILLNESS PERCEPTION IN SYSTEMIC SCLEROSIS WITH PULMONARY INVOLVEMENT
}

Bruna Giusto Bunjes (Hospital das Clínicas da Faculdade de Medicina da USP, SAO PAULO, SP, Brasil), Glaucia Ferreira Abrahão (Hospital das Clínicas da Faculdade de Medicina da USP, São Paulo, SP, Brasil), Percival Degrava Sampaio-Barros (Hospital das Clínicas da Faculdade de Medicina da USP, SAO PAULO, SP, Brasil), Ana Paula Luppino Assad (Hospital das Clínicas da Faculdade de Medicina da USP, SAO PAULO, SP, Brasil)

\section{BACKGROUND}

Lung disease is the current leading cause of death and the most common severe complication of systemic sclerosis (SSc). Due to its severity, we decided to focus on SSc patients with pulmonary involvement to determine their perception about the disease and correlate with quality of life, degree of disability and symptoms, as well as expectations.

\section{MATERIALS AND METHODS}

This cross-sectional study included SSc patients with symptomatic lung involvement. Clinical information were collected from an electronic register database. Revised Illness Perception Questionnaire (IPQR), 36Item Short Form Health Survey (SF-36), Health Assessment Questionnaire Disability index (HAQDI), COPD Assessment Test (CAT) and three direct questions regarding quality of life, expectations about treatment and survival were applied to patients. Correlations between questionnaires were made to evaluate patient perception measured by IPQR and if those perceptions would affect their opinion.

\section{RESULTS}

130 SSc patients, 55 (42\%) with limited SSc, 64 (49\%) diffuse and 11 (8\%) sine scleroderma. Mean age was $54 \pm 12.5$ years and mean disease duration $12 \pm 9$. All domains of IPQR statistically correlated with SF36, $\mathrm{HAQ}$ and CAT, except the perception of chronicity. The Pearson linear correlation was negative between SF36 and negative IPQR domains (meaning worse quality of life and higher negative perception); and positive between SF36 and positive domains (meaning higher quality of life and higher positive perception). Higher $\mathrm{HAQ}$ and CAT indicated higher disability and dyspnea, presenting a linear positive Pearson correlation $(p<0.0001)$.

A higher perception score was found comparing patients with moderate to severe disability $(\mathrm{HAQ}>1.25)$ or advanced dyspnea scores (CAT > 20) to patients with lower grade of disability or pulmonary symptoms. The relation between age, disease duration and IPQR had no statistical significance. Regarding the subjective opinion of the patients, established by direct questions, they presented optimistic answers as such $86.9 \%$ classified their quality of life as good, $45 \%$ expected cure or improvement with treatment in progress, and $85 \%$ believed that they would live more than 5 years. But no correlation between the IPQR domains and their opinion was found.

\section{CONCLUSION}

SSc patients with impaired quality of life, disability and major symptoms of dyspnea have higher perception of their disease by IPQR. However, the degree of perception does not appear to influence their opinion, suggesting an optimistic profile with more positive attitude and effective coping strategies. 